\title{
Photospheric magnetoconvection
}

\author{
Robert Cameron, Alexander Vögler and Manfred Schüssler \\ Max-Planck Instute for Solar System Research, Katlenburg-Lindau, Germany \\ cameron@mps.mpg.de
}

\begin{abstract}
Magnetic fields, convective motions and radiative energy transport all strongly influence each other in the solar photosphere. Attempts to understand, at a quantitative level, the diverse phenomona seen in the photosphere have relied on increasingly powerful computers since the 1980's. This approach has been remarkably successful and some recent examples, particularly cases where all three ingredients are important, will be discussed.
\end{abstract}

Keywords. Convection, magnetic fields, radiative transfer, Sun: photosphere

\section{Introduction}

The solar atmosphere changes from opaque to transparent over a few hundred kilometers (at least for a wide range of wavelengths). For this reason it is a challenging but rewarding region to understand and simulate. The challenge come from the fact that the physical conditions vary significantly: for example just below the photosphere convection is the dominant form of energy transport whilst a few hundred kilometers higher it is radiation. Further complications arise because the temperatures are such that the state of ionization of the gas is also changing (which is most important in terms of the energy it carries and therefore mainly affects the equation of state). Another complication comes from magnetic fields, which can strongly affect the temperature (through inhibition of the convection), the density (through partial evacuation of flux tubes) and the velocity field (via the Lorentz force). The major simplifications which are (usually) justifiable in the photosphere are that those underlying MHD and the assumption of local thermal equilibrium (LTE).

Realistic photospheric simulations thus need to include MHD, radiation, and an equation of state including the effects of partial ionization, usually in a fully 3 dimensional setting. Including these physical effects allows a direct comparison against, and a deeper understanding of, observations, e.g. see Khomenko et al. (2005a, b).

Before setting out the equations in section 2, and some simulation results in section 3 , we show in Figure 1 an image of a small part of the photosphere. This image illustrates several different types of photospheric features, sunspot umbra, pores, the quiet Sun and plage. All of these types of regions have recently been studied: realistic quiet Sun simulations by several authors beginning with Nordlund (1982), Nordlund (1983) up to Stein \& Nordlund (2006); umbral simulations by Schüssler \& Vögler (2006); plage studies, by eg Keller et al. (2004) and Carlsson et al. (2004); investigations of flux emergence through the photosphere Cheung (2006); and micropores by Bercik et al. (2003) and Vögler et al. (2005a). Realistic photospheric simulations of pores, partly described in Cameron et al. (2004) and Cameron et al. (2005), and of mixed polarity regions are discussed in more detail below. The approach has also been extended to the chromosphere by Wedemeyer et al. (2004). 


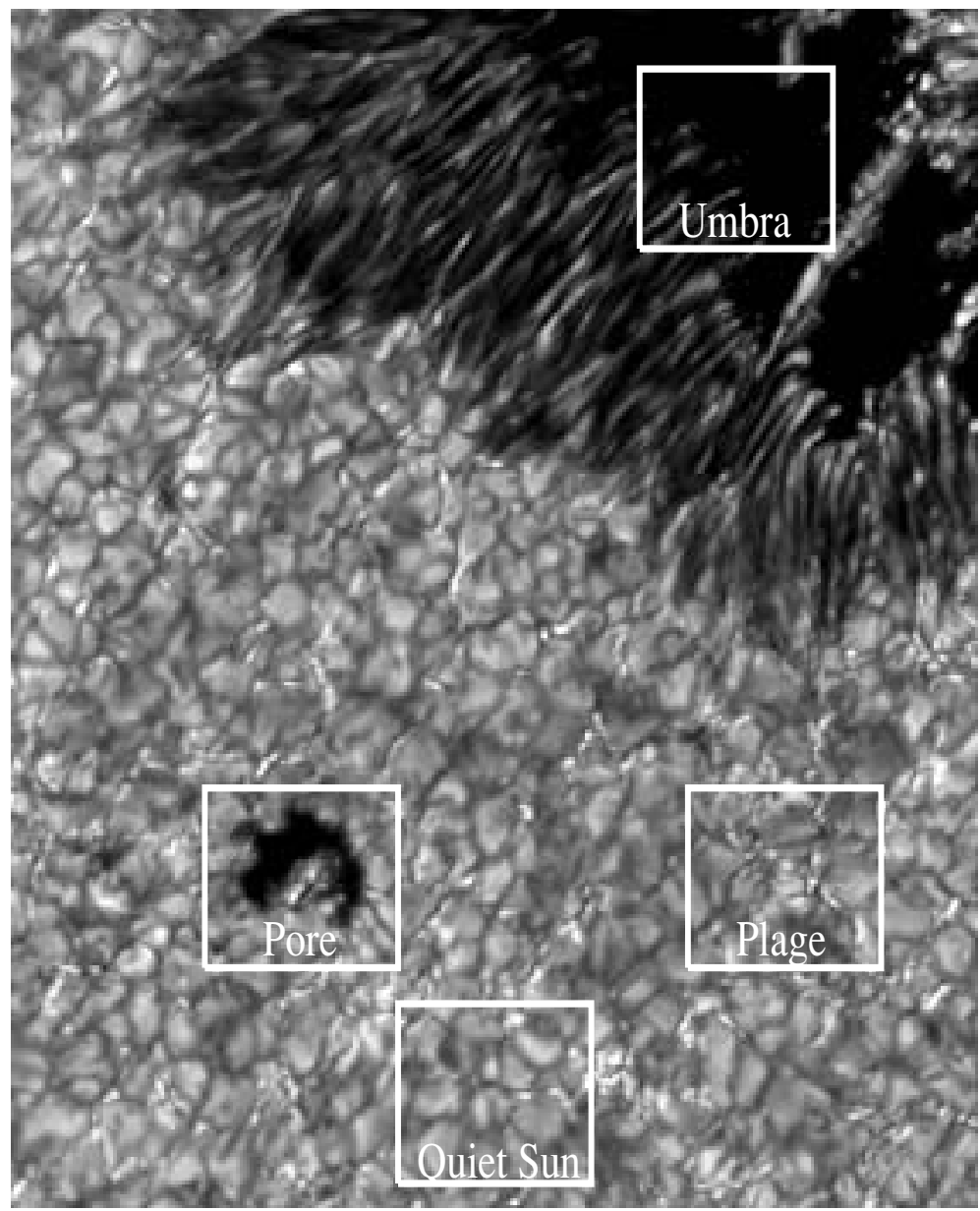

Figure 1. This figure shows a high resolution picture of the part of the solar surface observed in the broad "G-Band" of carbon monoxide. The image, which is from the KIS/VTT Obs. del Teide, Tenerife, and has been produced in this form by Markus Sailer, Göttingen University. Several types of regions which have recently been studied using numerical simulations are shown in this image.

\section{Formulation of the problem}

The equations and numerical methods, and some of the tests the code was subjected to are described in full in Vögler et al. (2005b). Here we content ourselves with a brief summary. The primary variables are: $\rho$ the density, $\rho \mathbf{U}$ the momentum, $\mathbf{B}$ the magnetic field and $e$ the total energy density per unit volume. The evolution equations are:

$$
\begin{aligned}
& \frac{\partial \rho}{\partial t}+\nabla \cdot(\rho \mathbf{U})=0 \\
& \frac{\partial \rho \mathbf{U}}{\partial t}+\nabla \cdot\left[\rho \mathbf{U U}+\left(p+\frac{|\mathbf{B}|^{2}}{8 \pi}\right) \underline{\mathbf{I}}-\frac{\mathbf{B B}}{4 \pi}\right]=\rho \mathbf{g}+\nabla \cdot \underline{\tau} \\
& \frac{\partial \mathbf{B}}{\partial t}+\nabla \cdot(\mathbf{U B}-\mathbf{B U})=-\nabla \times(\eta \nabla \times \mathbf{B})
\end{aligned}
$$




$$
\begin{aligned}
\frac{\partial e}{\partial t}+ & \nabla \cdot\left[\mathbf{U}\left(e+p+\frac{|\mathbf{B}|^{2}}{8 \pi}\right)-\frac{1}{4 \pi} \mathbf{B}(\mathbf{U} \cdot \mathbf{B})\right]=-\nabla \cdot[\mathbf{B} \times(\eta \nabla \times \mathbf{B})] \\
& +\nabla \cdot(\mathbf{U} \cdot \underline{\tau})+\nabla \cdot(K \nabla T)+\rho \mathbf{g} \cdot \mathbf{U}+Q_{\mathrm{rad}}
\end{aligned}
$$

where $Q_{\text {rad }}$ is the radiative heating, $p$ is the plasma pressure and $T$ is the temperature. These are calculated from the primary quantities using

$$
I_{\nu}\left(\tau_{\nu}, \boldsymbol{\mu}\right)=I_{\nu}(0) e^{-\tau_{\nu}}+\int_{0}^{\tau_{\nu}} B_{\nu}(\tau) e^{-\left(\tau_{\nu}-\tau\right)} d \tau
$$

where the integration is performed in the direction of $\boldsymbol{\mu}$,

$$
\begin{aligned}
J_{\nu} & =\frac{1}{4 \pi} \oint_{4 \pi} I_{\nu}(\boldsymbol{\mu}) d \Omega \\
Q_{\mathrm{rad}} & =4 \pi \rho \int_{4 \pi} \kappa_{\nu}\left(J_{\nu}-B_{\nu}\right) d \nu \\
\epsilon & =e-\rho \frac{|\mathbf{U}|^{2}}{2}-\frac{|\mathbf{B}|^{2}}{8 \pi} \\
p & =p(\epsilon, \rho) \\
T & =T(\epsilon, \rho)
\end{aligned}
$$

where $\tau_{\nu}$ is the optical depth at a particular wavelength $\nu$, and $p(\epsilon, \rho)$ and $p(\epsilon, \rho)$ are obtained from tables based on the Saha equations. The integration with respect to optical depth is performed using the method of short characteristics, and the integration over the solid angle $\Omega$ is performed using a number of fixed representative directions.

\subsection{Boundary conditions}

The boundary conditions are described in detail with in Vögler et al. (2005b), and are only briefly spelt out here for completeness. The bottom boundary condition is "open", that is material is allowed to freely enter or leave the computational domain. In regions of outflow the flow below the boundary is assumed to be that obtained by extrapolating from within the computation domain. In inflow regions the velocity is assumed to be vertical. The imposed uniform pressure at the bottom boundary is continually adjusted so that the mass within the domain is kept constant; and the energy per unit mass adjusted so that the time averaged over sufficiently energy flux associated with the radiation leaving the box matches the observed level of the real Sun. The magnetic field at the bottom boundary is assumed to be vertical.

The top boundary condition is closed, at least in the simulations described in this paper. This is to say that there are no flows across the top boundary. The magnetic field there is either vertical or matched to a potential field above.

\subsection{Diffusivities and Prandtl numbers}

This type of simulation aims to as closely as possible simulate the Sun. Since the value of the molecular viscosity is far below what we can hope to simulate, we adopt the approach of using an artifical viscosity which acts on the smallest scales, and steepest gradients, to stablize the scheme but has little direct effect on the larger well resolved scales.

In the context of realistic photospheric transfer the Prandtl number, the ratio of the viscous to thermal diffusivities, is conceptually problematic since above $\tau \approx 1$ radiative transfer is intrinsically nonlocal and not equivalent to thermal diffusion. The magnetic Prandtl number, the ratio of the viscous and magnetic diffusivity is problematic for a different reason: since we use a scale and gradient dependent artificial viscosity as 

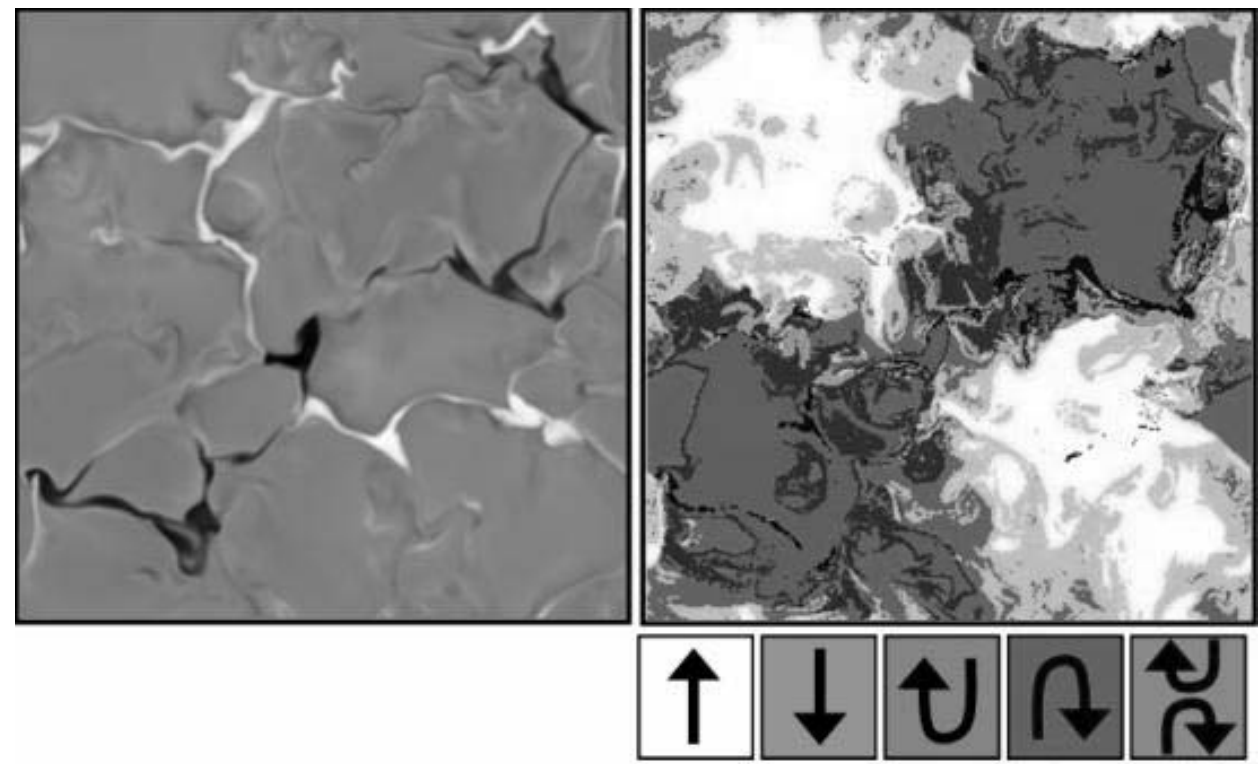

Figure 2. The magnetic field (left panel) and magnetic topology (right panel) at fixed geometric height near the average $\tau=1$ surface. These are from a snapshot of a mixed-polarity simulation. The light grey regions indicate regions where reconnection has created $\mathrm{U}$ and inverted $\mathrm{U}$ loops.

against the standard form of magnetic diffusivity, the ratio of the two depends on both the length scale and the gradients present in the flow. Away from shocks the magnetic Prandtl number should be on the correct side of unity, but more is difficult to say.

\section{Results: Mixed polarity simulations}

(The following discussion is based on work being prepared for publication by Vögler, Cameron and Schüssler.)

We here discuss the way in which opposite polarity patches reconnect and are removed from the observable layers of the Sun. The simulations for this set of experiments cover $6 \mathrm{Mm}$ in each of the horizontal directions and $1.4 \mathrm{Mm}$ in the vertical. They begin with a snapshot of fully developed convection on which a checker-board pattern of positive and negative vertical magnetic field is superposed. In the example shown in Figure 2 the simulation was began with 2 squares of positive flux and two squares of negative flux (a $2 \times 2$ initial geometry). The figure shows the magnetic field and magnetic topology a short time after the simulation was started. Whilst the magnetic field has been swept to the downflow lanes, the original $2 \times 2$ configuration is still clearly visible.

The initial phase of the evolution of the system is shown in Figure 3 with a $2 \times 1$ (ie one rectangle with field directed upwards, one with field directed downwards) initial condition for comparison. The exponential decay is consistent with a simple turbulent diffusivity according to a simple estimate based on a random walk with velocity and spatial scales close to those of the granules. This turbulent diffusivity is around 15 times larger than the included explicit diffusivity.

\section{Results: Pores}

(The following discussion is based on work being prepared for publication by Cameron, Vögler and Schüssler.) 


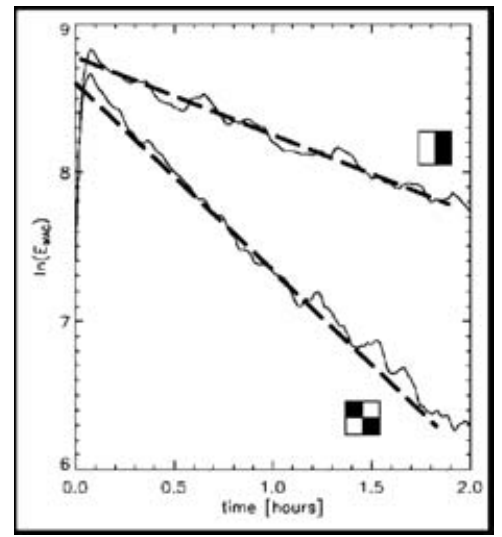

Figure 3. Time evolution of the magnetic energy of two mixed polarity simulations with different magnetic initial conditions. Since there is no net imposed flux the magnetic field decays freely. The decay rates are consistent with a random walk with granule-like parameters.
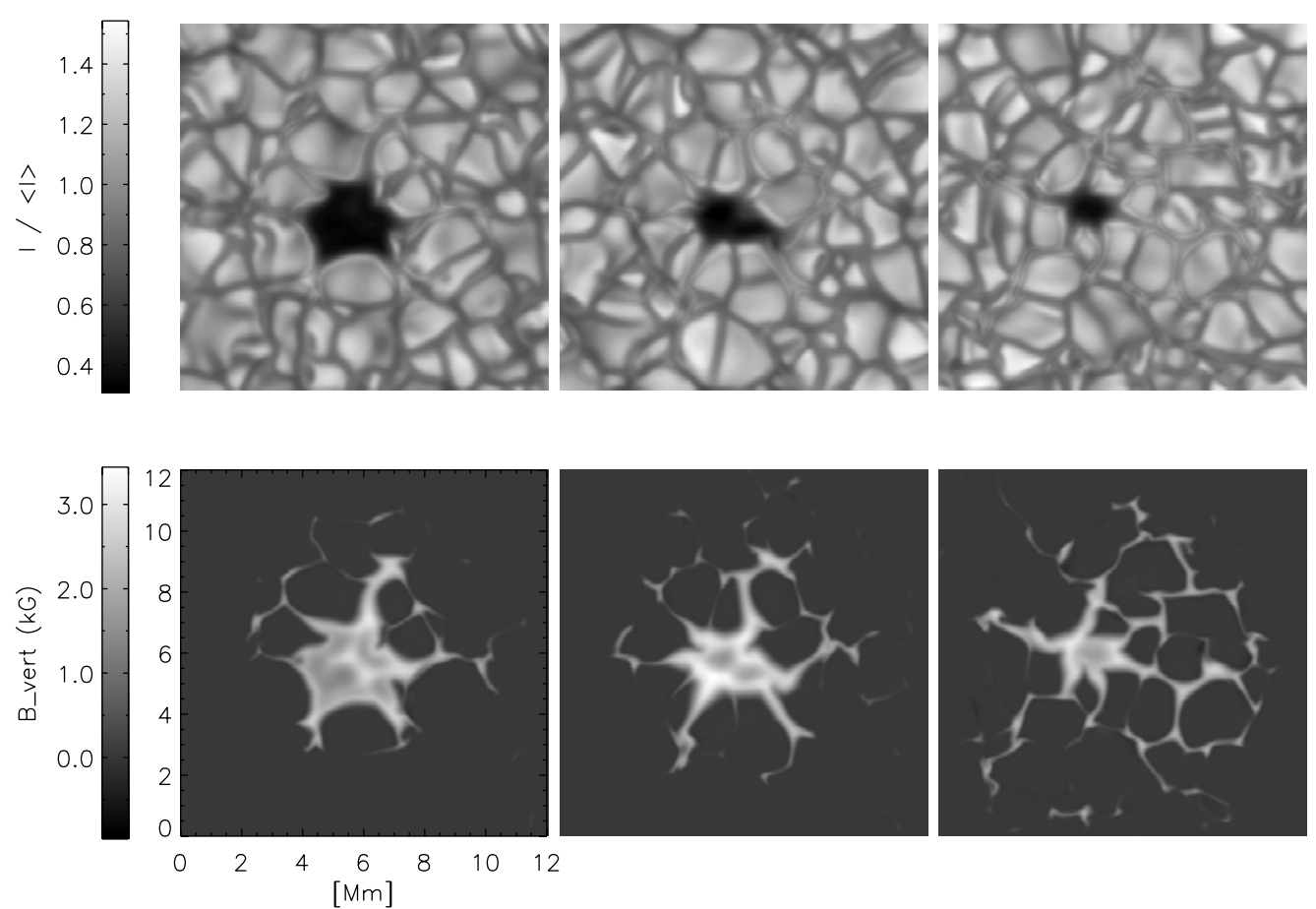

Figure 4. Evolution of a simulated pore over a 1 hour period. The top panel shows the emergent intensity, the bottom panel the vertical component of the magnetic field.

In this simulation we have modeled a solar pore. The simulation box is $12 \mathrm{Mm}$ in each of the horizontal directions and $1.4 \mathrm{Mm}$ in the vertical. A potential magnetic boundary condition is used at the top, and the temperature of gas entering through the bottom boundary has been reduced by $15 \%$ in regions where the magnetic field excedes $80 \mathrm{G}$. Since pores (as distinct from micropores) do not spontaneously form in photospheric 

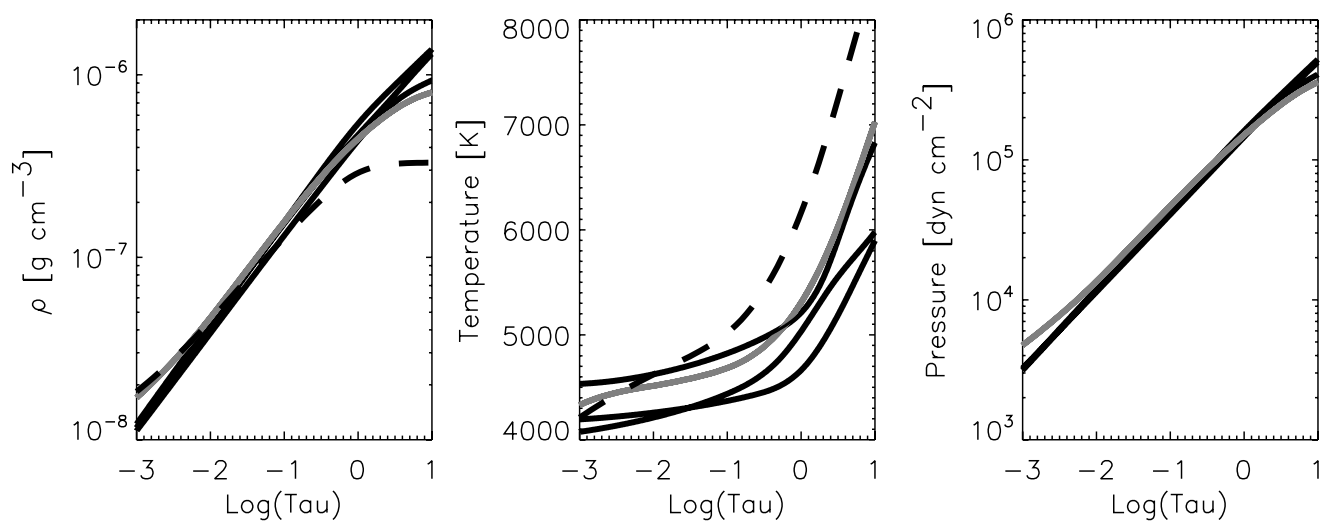

Figure 5. Comparison of the average vertical structure in the simulated pore (grey curve) and profiles derived by Sütterlin (1998) from observations. The dashed curve corresponds to the non-pore regions in the simulation.

simulations (Bercik et al., 2003; Vögler et al., 2005), we have had to impose them as an initial condition. The initial condition was generated by imposing a strong plug of flux in a 2-D simulation and waiting for the system to reach a quasi-static equilibrium. The advantage of this approach is that the structure is much longer lived in 2-D then in 3-D. This is due to differences in topology: in 2-D there are granules at both edges of the pore, in 3 -D there are intergranular lanes perpendicular to the edge of the pore along which flux can escape as can be seen in Figure 4.

The time evolution over a period of 1 hour is shown in Figure 4 . The pore decays as flux escapes through intergranular lanes connected to the pore. Figure 5 shows that the model successfully reproduces the observed vertical structure of the pores. The comparison is of a pore from about half-way through the simulation. For most of the time the simulation was run with grey radiative transfer, however for this comparison the simulation was run for several (solar) minutes using 4-bin opacities, see Vögler et al. (2005b). The observational analysis is that of Sütterlin (1998), and the comparison is best for the smallest pore he studied: which is approximately the same size as that of the simulation at the time of comparison. This is an encouraging result which suggest that, like granulation, the surface appearance of pores is dominated by surface effects.

\section{References}

Bercik, D.J., Nordlund, A. \& Stein, R.F. 2003, GONG+ 2002. Local and Global Helioseismology: the Present and Future (ESA) SP-517 p. 201

Carlsson, M., Stein, R.F., Nordlund, Å \& Scharmer, G.B. 2004, Ap.J. 610, L137

Cameron, R., Vögler, A., Schüssler, M. \& Zakharov, V. 2005, 11th European Solar Physics Meeting. The dynamic Sun: Challenges for theory and observation (ESA) SP-600

Cameron, R., Vögler, A., Shelyag, S., \& Schüssler, M. 2004, in: Sakurai, T. \& Sekii, T. (eds) The Solar-B mission and the forefront of solar physics: The fifth Solar-B science meeting (ASP) 57

Cheung, M., 2006, PhD thesis, University of Göttingen

Keller, C.U., Schüssler, M., Vögler, A. \& Zakharov, V. 2004, Ap.J., 607, L59

Khomenko, E. V., Martínez González, M. J., Collados, M., Vögler, A., Solanki, S. K., Ruiz Cobo, B. \& Beck, C., 2005, A\&3A, 436, L27

Khomenko, E. V., Shelyag, S., Solanki, S. K. \& Vögler, A., 2005 A $\& A, 442,1059$ 
Nordlund, A. 1982, A\& A 107, 1

Nordlund, Å. 1982, in: Stenflo, J.O. (ed.) IAU Symp. 102. Solar and Stellar Magnetic fields: Origins and Coronal effects. 79

Schüssler, M., Shelyag, S., Berdyugina, S., Vögler, A. \& Solanki, S.K., 2003 Ap.J (Academic), 597, L173

Schüssler, M. \& Vögler, A., 2006 ApJ, 641, L73

Stein, R. F. \& Nordlund, Å., 2006 ApJ 642, 1246

Sütterlin, P. 1998, A\&A 333, 305

Vögler, Shelyag, Schüssler, 2005, A\&\&A 429, 335

Vögler, A., Shelyag, S., Schüssler, M., Cattaneo, F., Emonet, T. \& Linde, T., 2005 A\&A, 429, 335

Wedemeyer, S., Freytag, B., Steffen, M., Ludwig, H.-G. \& Holweger, H., 2004, A\&\&A, 414, 1121

\section{Discussion}

STEIN: This is very beautiful work. If you don't want to wait for the papers in preparation, some of these results will appear shortly in the proceedings of the November 2005 Solar $\mathrm{B}$ meeting in the review of solar magneto-convection I gave.

BRUN: How are your simulations of pores, umbrae, etc affected by small scale dynamo action versus the cases that you showed us that have their B-field decaying?

Cameron: Their is an imposed field in the umbrae and pore simulations which cannot decay, the magnetic flux through the box being a conserved quantity. This component is likely to dominate that produced by a local dynamo. The simulation of the mixed polarity region on the otherhannd has zero net flux passing through the box and so is free to decay. With the parameters used here the field decays over the length of the simulation. One of us (Alexander Vögler) is currently exploring what happens in parameter regimes where dynamo action occurs.

ToOmRE: Please clarify the magnetic conditions and general boundary conditions for flows and fields in your simulation

Cameron: These are naturally important issues. They will be/are better described in the printed version of this talk. 